\title{
CARACTERIZACIÓN DE Escherichia coli D7111 PRODUCTORA DE $\beta$-LACTAMASA TEM-176
}

\author{
Joaquim Ruiz@1,a, Maria J. Pons@1,a, Susan Mosquito@2,a, Theresa J. Ochoa®2,3, \\ Yolanda Sáenz 40 \\ 1 Laboratorio de Genética Molecular y Bioquímica, Universidad Científica del Sur, Lima, Perú. \\ 2 Instituto de Medicina Tropical «Alexander von Humboldt», Universidad Peruana Cayetano Heredia, Lima, Perú. \\ 3 School of Public Health, University of Texas Health Science Center at Houston, Houston, Estados Unidos. \\ 4 Área de Microbiología Molecular, Centro de Investigación Biomédica de La Rioja, Logroño, España. \\ a $\mathrm{PhD}$ en Biología; ${ }^{\mathrm{b}}$ médico infectóloga pediatra, $\mathrm{PhD}$ en Medicina; ${ }^{\mathrm{c}} \mathrm{PhD}$ en Bioquímica y Biología Molecular y Celular.
}

\section{RESUMEN}

El presente reporte es la descripción original de $b l a_{\mathrm{TEM}-176}$. Se caracterizaron los mecanismos de resistencia a antimicrobianos de un aislamiento de Escherichia coli enterotoxigénica, determinándose la resistencia a 22 antimicrobianos categorizados en 15 grupos diferentes mediante difusión en agar, estableciéndose grupo filogenético, mecanismos de resistencia y presencia de integrones de Clase 1 y 2 mediante PCR. Integrones y genes de resistencia a $\beta$-lactámicos fueron secuenciados. El aislamiento del grupo filogenético $\mathrm{A}$, mostró resistencia o sensibilidad disminuida a ampicilina, amoxicilina más ácido clavulánico, ácido nalidíxico, ciprofloxacino, estreptomicina, kanamicina, tetraciclina, trimetoprim, sulfisoxazol, cotrimoxazol, azitromicina y nitrofurantoina, detectándose la presencia de $b l a_{\mathrm{TEM}}$, aadA1/2, aphA1, sul3, tet(A) y un integron de Clase 2 conteniendo un gen $d f r A 1$. La resistencia a quinolonas se relacionó con la substitución $\mathrm{Ser}_{83}$ Ala. La secuencia de TEM mostró la substitución Ala $_{222}$ Val, la cual a la fecha no había sido descrita, reportándose como una nueva $\beta$-lactamasa, con el nombre de $b l a_{\text {TEM-176. }}$.

Palabras clave: Ampicilina; Amoxicilina-Ácido Clavulánico; beta-Lactamasas; Resistencia a Antibióticos; Escherichia coli Enterotoxigénica; Epidemiología Molecular; Integrones (fuente: DeCS BIREME).

\section{CHARACTERIZATION OF Escherichia coli D7111 PRODUCING THE $\boldsymbol{\beta}$-LACTAMASE TEM-176}

Citar como: Ruiz J, Pons MJ, Mosquito S, Ochoa TJ, Sáenz Y. Caracterización de Escherichia coli D7111 productora de $\beta$-lactamasa TEM-176. Rev Peru Med Exp Salud Publica. 2021;38(1):13035. doi: https://doi.org/10.17843/ rpmesp.2021.381.6727.

Correspondencia: Joaquim Ruiz, Laboratorio de Genética Molecular y Bioquímica, Universidad Científica del Sur, Panamericana Sur Km 19, Lima, Peru; joruiz.trabajo@gmail.com

Recibido: 18/08/2020 Aprobado: 06/01/2021 En línea: 03/02/2021

\begin{abstract}
The present report is the original description of $b l a_{\text {TEM-176. }}$ The mechanisms of resistance to antimicrobial agents were determined in an enterotoxigenic Escherichia coli, determining the susceptibility to 22 antimicrobials classified in 15 different groups by agar diffusion and establishing the phylogenetic group, mechanisms of resistance and presence of Class 1 and 2 integrons. Integrons and $\beta$-lactam resistance genes were sequenced. The isolate, belonging to phylogenetic group $\mathrm{A}$, showed the presence of resistance or diminished susceptibility to a ampicillin, amoxicillin plus clavulanic acid, nalidíxic acid, ciprofloxacin, streptomycin, kanamycin, tetracycline, trimethoprim, sulfisoxazole, cotrimoxazole, azithromycin and nitrofurantoin, carrying $b l a_{\text {TEM }}, \operatorname{aad} A 1 / 2, a p h A 1, \operatorname{sul} 3$, tet(A) and a Class 2 integron containing a $d f r A 1$ gene. Quinolone resistance was related to the substitution $\operatorname{Ser}_{83}$ Ala. The TEM sequencing showed the presence of the new substitution $\mathrm{Ala}_{222} \mathrm{Val}$, which led to the description of the new $\beta$-lactamase $b l a_{\text {TEM-176. }}$
\end{abstract}

Keywords: Ampicillin; Amoxicillin-Clavulanic Acid; beta-Lactamases; Antibacterial Drug Resistance; Enterotoxigenic Escherichia coli; Molecular Epidemiology; Integrons (source: MeSH NLM).

\section{INTRODUCCIÓN}

La familia de los $\beta$-lactámicos está entre las familias de antibióticos más ampliamente utilizadas a nivel mundial, tanto en salud humana como veterinaria. No obstante, el desarrollo de 
microorganismos resistentes a estos antibióticos compromete su utilidad. Así, en algunas zonas, incluyendo diferentes regiones de Perú, los niveles de resistencia a los $\beta$-lactámicos más antiguos, como ampicilina bordea o supera el $90 \%{ }^{(1,2)}$. Mientras, los niveles de resistencia a $\beta$-lactámicos más modernos, como cefalosporinas de $3^{\text {a }}$ y $4^{\text {a }}$ generación se sitúan alrededor del $50 \%{ }^{(3)}$.

En gramnegativos el fenómeno de la resistencia a $\beta$-lactámicos se asocia principalmente a la producción de $\beta$-lactamasas. Entre estas, destacan por su elevada frecuencia las $\beta$-lactamasas tipo TEM, las cuales han sido profusamente reportadas en Perú ${ }^{(2,3)}$, aunque en la mayoría de las ocasiones sólo se ha determinado su presencia, sin desarrollar estudios de secuenciación que permitan determinar variantes.

En este contexto, en 2007, durante un estudio sobre diarrea infantil y análisis de resistencia a antimicrobianos realizado en Lima, se aisló la cepa diarreogénica (enterotoxigénica) de Escherichia coli D7111 (1), la cual se decidió caracterizar molecularmente, identificando la variante de gen $b l a_{\text {TEM }}$ que presentaba. Por lo tanto, este reporte tiene el objetivo de caracterizar molecularmente la cepa de E. coli D7111 incluyendo la descripción original del gen $b l a_{\text {TEM-176 }}$.

\section{EL ESTUDIO}

La cepa de E. coli D7111 fue aislada en el curso de un estudio de cohorte prospectivo pasivo desarrollado en zonas periurbanas del sur de Lima que incluyó a 1034 niños de entre 2 y 12 meses de edad ${ }^{(1)}$.

Mediante difusión en agar ${ }^{(4)}$, se determinó la sensibilidad a 22 antimicrobianos categorizados en 15 grupos. Así, se determinó la resistencia a penicilinas (ampicilina), penicilinas + inhibidores de $\beta$-lactamasas (amoxicilina más ácido clavulánico [AMC]), cefamicinas (cefoxitina), cefalosporinas de espectro extendido (cefotaxima, ceftazidima, cefepime), monobactams (aztreonam), carbapenemes (imipenem), aminoglucósidos (amikacina, gentamicina, kanamicina), estreptomicinas (estreptomicina), anfenicoles (cloranfenicol), quinolonas (ácido nalidíxico), fluoroquinolonas (ciprofloxacino, norfloxacino), inhibidores de folato (trimetoprim, cotrimoxazol), macrólidos (azitromicina) tetraciclinas (tetraciclina), acidos fosfónicos (fosfomicina), nitrofuranos (nitrofurantoina) y sulfamidas (sulfisoxazol). Los resultados se interpretaron de acuerdo con la guía de 2010 del Clinical and Laboratory Standards Institute (CLSI) ${ }^{(4)}$.

La presencia de $\beta$-lactamasas de espectro extendido (BLEE) se analizó mediante el test de sinergia de doble disco ${ }^{(3)}$, mientras que la de AmpC plasmídicas ( $\mathrm{pAmpC}$ ) se determinó mediante comparación de los diámetros de inhibición del disco de cefoxitina en presencia o ausencia de $200 \mu \mathrm{g}$ de cloxacilina ${ }^{(5)}$.

La presencia de $\beta$-lactamasas tipo CARB, OXA-1-like, OXA-2-like, OXA-5-like, SHV y TEM, se determinó por PCR (de las siglas en inglés de Polymerase Chain Reaction)

\section{MENSAJES CLAVE}

Motivación para realizar el estudio: Si bien en diversos estudios en Perú se establece la presencia de $b l a_{\mathrm{TEM}}$, existe una escasez de datos sobre las variantes circulantes.

Principales hallazgos: Un gen $b l a_{\text {TEM }}$ de Escherichia coli fue secuenciado, presentando una única variación, conducente al cambio $\mathrm{Ala}_{222} \mathrm{Val}$, con respecto a TEM-1. En la fecha de desarrollo del estudio (2010) la secuencia obtenida no había sido descrita, adjudicándosele el nombre de bla $a_{\text {TEM-176 }}$.

Implicancias: La detección de una nueva $\beta$-lactamasa, manifiesta la presencia de genes de resistencia no descritos en el área, sugiriendo el potencial de secuenciar genes íntegros cuando fuere posible.

(Tabla 1). Los productos positivos fueron recuperados, purificados y enviados a Macrogen (Seul, Corea del Sur) para ser secuenciados en ambos sentidos.

Los grupos filogenéticos se establecieron por PCR (amplificación simultánea de $\operatorname{chuA,~yjaA~y~tspE4C2).~Asimismo~se~}$ estableció la presencia de los genes aadA1/2, aphA1 (aph(3')Ia) y aphA2 ( $a p h\left(3^{\prime}\right)$-IIa) implicados en resistencia a estreptomicinas y aminoglucósidos; tet(A) y tet(B) implicados en resistencia a tetraciclinas; $d f r A 1, d f r A 5, d f r A 6, d f r A 7, d f r A 12$, $d f r A 13, d f r A 14, d f r A 15, d f r A 15 b, d f r A 16, d f r A 16 b, d f r A 17$ relacionados con desarrollo de resistencia a trimetoprim y sul1, sul2 y sul3 causantes de resistencia a sulfamidas. Adicionalmente se determinó la presencia de integrones Clase 1 y Clase 2 por PCR, así como se amplificó la región determinante de resistencia a quinolonas de los genes gyrA y parC (Tabla 1). En el caso de los genes $d f r$, se identificó la variante presente mediante RFLP (de las siglas en inglés de Restriction Lenght Polymorphism) de los amplicones obtenidos según la metodología previamente descrita ${ }^{(6,7)}$. Las mutaciones en gyrA y parC así como los productos positivos para integrones fueron caracterizados mediante secuenciación.

Información sobre las secuencias de referencia (DNA/ Aminoácidos) de los genes de resistencia considerados en este estudio (así como de otros) se puede encontrar en la página https://www.ncbi.nlm.nih.gov/refseq/.

El estudio original fue aprobado por el Comité de Ética del Instituto de Investigación Nutricional con el código 2462006/CEI-IIN.

\section{HALLAZGOS}

La cepa E. coli D7111, identificada como perteneciente al filogrupo A, presentó resistencia a ampicilina, AMC, azitromicina, cotrimoxazol, kanamicina, sulfisoxazol, tetraciclina, 
Tabla 1. Cebadores utilizados en este estudio.

\begin{tabular}{|c|c|c|c|c|c|}
\hline Gen & Cebador 1 & Cebador 2 & pb & ${ }^{\circ} \mathrm{C}$ & Ref \\
\hline$b l a_{\mathrm{TEM}}$ & ATTCTTGAAGACGAAAGGGC & ACGCTCAGTGGAACGAAAAC & 1150 & 60 & 3 \\
\hline$b l a_{\mathrm{SHV}}$ & ATGCGTTATATTCGCCTGTG & TTAGCGTTGCCAGTGCTCG & 841 & 55 & 3 \\
\hline$b l a_{\text {CARB }}$ & AATGGCAATCAGCGCTTC & GGGGCTTGATGCTCACT & 586 & 56 & 3 \\
\hline$b l a_{\text {OXA-1-LIKE }}$ & ACCAGATTCAACTTTCAA & TCTTGGCTTTTATGCTTG & 598 & 55 & 3 \\
\hline$b l a_{\mathrm{OXA}-2-\mathrm{LIKE}}$ & CGATAGTTGTGGCAGACGAA & ССАСТСААСССАТССТАССС & 550 & 55 & 3 \\
\hline$b l a_{\mathrm{OXA}-5-\mathrm{LIKE}}$ & TATATTCCAGCATCAACATT & ATGATGCCCTCACTTGCCAT & 605 & 55 & 3 \\
\hline gyrA & AAATCTGCCCGTGTCGTTGGT & GCCATACCTACGGCGATACC & 343 & 55 & 3 \\
\hline $\operatorname{parC}$ & AAACCTGTTCAGCGCCGCATT & GTGGTGCCGTTAAGCAAA & 395 & 59 & 3 \\
\hline aphAl & ATGGGCTCGCGATAATGTC & CTCACCGAGGCAGTTCCAT & 600 & 50 & 6 \\
\hline aphA2 & GAACAAGATGGATTGCACGC & GCTCTTCAGCAATATCACGG & 680 & 50 & 6 \\
\hline aadA1, aadA2 & GCAGCGCAATGACATTCTTG & ATCCTTCGGCGCGATTTTG & 282 & 60 & 6 \\
\hline $\operatorname{tet}(\mathrm{A})$ & GTAATTCTGAGCACTGTCGC & CTGCCTGGACAACATTGCTT & 937 & 62 & 6 \\
\hline $\operatorname{tet}(\mathrm{B})$ & CTCAGTATTCCAAGCCTTTG & CTAAGCACTTGTCTCCTGTT & 416 & 57 & 6 \\
\hline$d f r A 1, d f r 5, d f r 15, d f r 15 b, d f r 16, d f r 16 b$ & GTGAAACTATCACTAATGG & TTAACCCTTTTGCCAGATTT & 474 & 55 & 6 \\
\hline$d f r A 6, d f r 14$ & GAGCAGCTICTITTIAAAGC & TTAGCCСTTTIICCAATTTT & 393 & 60 & 6 \\
\hline$d f r A 7, d f r 17$ & TTGAAAATTTCATTGATT & TTAGCCTTTTTTCCAAATCT & 474 & 55 & 6 \\
\hline$d f r A 12, d f r 13$ & GGTGSGCAGAAGATTTTTCGC & TGGGAAGAAGGCGTCACCСTC & 319 & 60 & 6 \\
\hline sul1 & TGGTGACGGTGTTCGGCATTC & GCGAGGGTTTCCGAGAAGGTG & 789 & 63 & 6 \\
\hline sul2 & CGGCATCGTCAACATAACC & GTGTGCGGATGAAGTCAG & 722 & 50 & 6 \\
\hline sul3 & CATTCTAGAAAACAGTCGTAGTTCG & CATCTGCAGCTAACCTAGGGCTTTGGA & 990 & 51 & 6 \\
\hline RV1 & GGCATCCAAGCAGCAAG & AAGCAGACTTGACCTGA & Var. & 55 & 6 \\
\hline RV2 & CGGGATCCCGGACGGCATGCACGATTTGTA & GATGCCATCGCAAGTACGAG & Var. & 60 & 6 \\
\hline chuA & GACGAACCAACGGTCAGGAT & TGCCGCCAGTACCAAAGACA & 279 & 59 & 7 \\
\hline yjaA & TGAAGTGTCAGGAGACGCTG & ATGGAGAATGCGTTCCTCAAC- & 211 & 59 & 7 \\
\hline tspE4C2 & GAGTAATGTCGGGGCATTCA & CGCGCCAACAAAGTATTACG & 152 & 59 & 7 \\
\hline
\end{tabular}

pb: Tamaño de los amplicones en pares de bases; ${ }^{\circ} \mathrm{C}$ : Temperatura de anillamiento; Ref: Referencia; RV: Región Variable (1 - de integrones de Clase 1; 2 - de integrones de Clase 2); Var: Variable

trimetoprim y ácido nalidíxico, así como sensibilidad intermedia a estreptomicina, nitrofurantoina y ciprofloxacino (Tabla 2). En el caso de azitromicina, el halo de inhibición fue de $<6 \mathrm{~mm}$, por lo que aun en ausencia de punto de corte establecido en CLSI, se clasificó como resistente.

El estudio fenotípico de $\beta$-lactamasas fue negativo, tanto para BLEE como para $\mathrm{pAmpC}$, siendo $b l a_{\mathrm{TEM}}$ el único gen codificante de $\beta$-lactamasas amplificado. El resultado de la secuenciación mostró la presencia de una diferencia a nivel de nucleótido $\left(\mathrm{A}_{665} \mathrm{~T}\right)$ respecto a la secuencia de $b l a_{\text {TEM-1 }}$, conducente al cambio aminoacídico $\mathrm{Ala}_{222} \mathrm{Val}$. Confrontada la secuencia aminoacídica con las descritas a la fecha, se comprobó su novedad, siendo sometida al repositorio correspondiente (www.lahey.com, actualmente transferido al National Center for Biotechnology Information - NCBI - https://www.ncbi.nlm.nih.gov/pathogens/submit-beta-lactamase/), siendo confirmada como una nueva variante de TEM, y recibió el nombre de TEM-176 (Figura 1). Consecuentemente, la secuencia se reportó al GenBank, y es accesible bajo los códigos: GU550123 - Secuencia DNA original; NG_050215 - Secuencia DNA de referencia; Secuencia Proteica de Referencia WP_038976851.

Adicionalmente, la cepa E. coli D7111 presentó los genes aadA1/2, aphA1 (codificante para la enzima modificante de aminoglucósidos, APH(3')-Ia), tet(A), dfrA1 y sul3 así como el cambio aminoacídico $\operatorname{Ser}_{83}$ Ala en GyrA. El estudio de integrones mostró la presencia de un integrón de clase 2, de aproximadamente $800 \mathrm{pb}$, conteniendo el casete génico dfrA1 (Tabla 2).

\section{DISCUSIÓN}

El presente trabajo reporta la presencia de una variante de TEM que, a la fecha de desarrollo del estudio, no había sido identificada por lo que, tras ser sometida a los responsables de mantenimiento de nomenclatura, recibió el nombre de TEM-176.

La familia de $\beta$-lactamasas tipo TEM fue descrita inicialmente en $1965^{(8)}$, contando en la actualidad con 190 variantes secuenciadas y presentes en https://www.ncbi.nlm.nih. gov/pathogens/refgene/\#gene_family:(blaTEM) que van de TEM-1 a TEM-242 (nótese la ausencia en la base de datos de NCBI de $\beta$-lactamasas tipo TEM con numeración asignada).

TEM-176 sólo difiere en un aminoácido con respecto a TEM-1. Aunque en apariencia insignificantes, diferencias de un aminoácido pueden implicar diferencias en el espectro de actividad (tanto sobre $\beta$-lactámicos existentes, como sobre $\beta$-lactámicos que se diseñen en momentos ulteriores a su descripción). Asimismo, esas diferencias pueden tener implicancias evolutivas, dado que la adquisición de una mutación puntual podría hacer evolucionar con mayor facilidad TEM-176 a otras variantes de TEM, inclusive BLEE, como sería el caso de TEM-147 (Secuencia Proteica de Referencia: 
Tabla 2. Perfil de resistencia y mecanismos subyacentes.

\begin{tabular}{|c|c|c|}
\hline Antimicrobiano & Sensibilidad & Mecanismo \\
\hline Ampicilina & $R$ & TEM-176 \\
\hline $\mathrm{AMC}$ & $\mathrm{R}$ & TEM-176? \\
\hline Cefoxitina & S & --- \\
\hline Cefotaxima & S & --- \\
\hline Ceftazidima & S & --- \\
\hline Cefepime & S & --- \\
\hline Aztreonam & S & -- \\
\hline Imipenem & S & \\
\hline Ácido Nalidixico & $\mathrm{R}$ & $\operatorname{Ser}_{83} \mathrm{Ala}(\mathrm{GyrA})$ \\
\hline Ciprofloxacino & I & $\operatorname{Ser}_{83} \mathrm{Ala}(\mathrm{GyrA})$ \\
\hline Norfloxacino & $S$ & $\operatorname{Ser}_{83} \mathrm{Ala}(\mathrm{Gyr} A)$ \\
\hline Amikacina & S & --- \\
\hline Gentamicina & S & --- \\
\hline Kanamicina & $\mathrm{R}$ & $\mathrm{APH}\left(3^{\prime}\right)-\mathrm{Ia}$ \\
\hline Estreptomicina & I & ANT(3")-I \\
\hline Azitromicina & $\mathrm{R}$ & ND \\
\hline Cloranfenicol & S & \\
\hline Trimetoprim & $\mathrm{R}$ & DfrA1 \\
\hline Sulfisoxazol & $\mathrm{R}$ & Sul3 \\
\hline Cotrimoxazol & $\mathrm{R}$ & DfrA $1+$ Sul3 \\
\hline Tetraciclina & $\mathrm{R}$ & $\operatorname{Tet}(\mathrm{A})$ \\
\hline Nitrofurantoina & I & ND \\
\hline Fosfomicina & S & \\
\hline
\end{tabular}

AMC: Amoxicilina más ácido clavulánico; S: Sensible; I: Intermedio; R: Resistente; ND: No determinado; ?: No establecido

WP_063864859), la cual presenta una sola diferencia aminoacídica con respecto a TEM-176, $\operatorname{Arg}_{162}$ His, en adición a la presencia de $\mathrm{Val}_{222}$.

Posteriormente a su detección en la cepa D7111 y su inclusión en la nomenclatura, TEM-176 ha sido descrita en diversas ocasiones. Así, en GenBank (https://www.ncbi.nlm. nih.gov/ipg/WP_038976851.1) consta su presencia en E. coli (29 cepas), Salmonella enterica (61 cepas, pertenecientes tanto a serotipos frecuentes como Typhimurium, Enteritidis o Hadar, o infrecuentes como Brancaster o Schwarzengrund), Klebsiella pneumoniae (2 cepas), Citrobacter amalonaticus (2 cepas), Citrobacter portucalensis (1 cepa) y Vibrio parahaemolyticus (2 cepas), siendo en la mayoría de los casos detectada en el curso de estudios de secuenciación genómica.

Probablemente la presencia de esta ( $u$ otras) variantes de $b l a_{\text {TЕM }}$ haya pasado desapercibida en numerosos estudios, dada la necesidad de secuenciar el amplicón de $b l a_{\text {TEM }}$ para poder efectuar la identificación correcta. A ello también puede haber contribuido el hecho de no afectar a cefalosporinas de $3^{\text {a }}$ y $4^{\text {a }}$ generación. No obstante, desde su descripción e introducción en GenBank el 31 de enero de 2010, $b l a_{\text {TEM-176 }}$

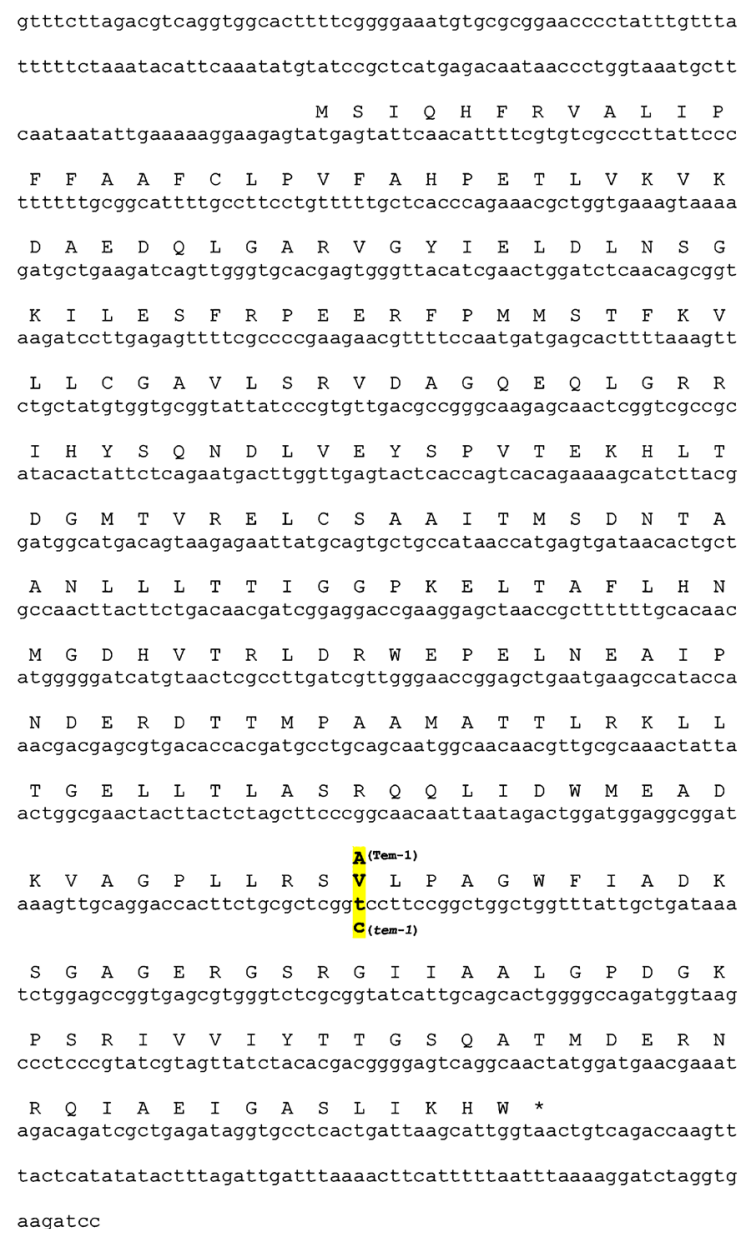

En letras minúsculas se encuentra la secuencia de un fragmento de ADN de 1043 pb que contiene el gen $b a_{a}$ (GenBank: GU550123), sobre ella está anotada en letras mayúsculas la secuencia aminoacídica de TEM-176. En negrita y resaltado en amarillo se encuentran las diferencias con respecto a TEM-1 (GenBank: NG_050145), aminoacídicas en la parte superior, nucleotídicas en la inferior.

Figura 1. Secuencia del gen blaTEM-176

ha sido reportada en microorganismos aislados de muestras de orígenes variados (ej: humanas, animales o alimentarias), y de localizaciones geográficas dispares, como por ejemplo Austria, Australia, Ecuador, Singapur o Tanzania, mostrando así una diseminación a nivel mundial ${ }^{(9-13)}$.

Si bien, hay escasos datos sobre su localización genómica, en algunos casos se ha comprobado su presencia en plásmidos del grupo IncX ${ }^{(10,11)}$, como pSGMCR103 (un plásmido del grupo IncX1), el cual, entre otros determinantes de resistencia, además de $b l a_{\text {TEM-176 }}$ transporta el gen $m c r-5.1^{(11)}$.

Adicionalmente, cabe reseñar que se ha detectado su presencia en estudios metagenómicos realizados en Perú ${ }^{(14)}$. Este hecho es interesante, ya que parecería que pese a la escasez de estudios encaminados a la secuenciación de $\beta$-lactamasas tipo TEM en Perú, la presencia de TEM-176 ha sido descrita por diferentes autores de manera independiente en el país, lo que sugiere que podría ser relativamente frecuente en el área. 
El hecho que la cepa D7111 sea resistente a AMC, junto con la no detección de otras $\beta$-lactamasas, abre la puerta a que este inhibidor de $\beta$-lactamasas no inhiba de manera completa a TEM-176, lo que permitiría categorizar a esta $\beta$-lactamasa como IRT (por las siglas en inglés de Inhibitor Resistant TEM). No obstante, este aspecto debería ser verificado de manera específica e inequívoca, dado que podrían coexistir $\beta$-lactamasas no identificadas, así como se ha constatado que la sobreexpresión de $\beta$-lactamasas tipo TEM, como por ejemplo TEM-1, puede conllevar sensibilidad disminuida o resistencia a AMC ${ }^{(15)}$.

La cepa D7111, asimismo presentó otros determinantes de resistencia, como es el caso del gen $a p h A 1$, subyacente tras la resistencia a kanamicina detectada ${ }^{(16)}$. APH(3')-Ia se ha descrito previamente en numerosos microorganismos, incluyendo todos los gramnegativos del grupo ESKAPE (Klebsiella pneumoniae, Acinetobacter baumannii, Pseudomonas aeruginosa y Enterobacter spp.) así como otros microorganismos causantes de diferentes patologías como Escherichia coli o Salmonella spp. (https://card.mcmaster.ca/ ontology/39041). Sin embargo, hasta donde sabemos, esta es la primera descripción de esta enzima en Perú. La ubicuidad de APH(3')-Ia junto con los elevados niveles de resistencia a kanamicina detectados en aislamientos clínicos en Perú hace que este hallazgo no sea sorprendente. Asimismo, se detectó la presencia de otro gen codificante de enzimas modificante de aminoglucósidos, un gen tipo aadA. Los genes aadA codifican nucleotidiltransferasas inactivantes de estreptomicina y espectinomicina ${ }^{(16)}$, encontrándose ampliamente diseminados a nivel mundial, aunque a diferencia del presente caso, a menudo asociados a integrones, tanto de Clase 1 como de Clase 2 (http://integrall.bio.ua.pt/).

En consonancia con lo descrito en la literatura, la substitución $\mathrm{Ala}_{83}$ en GyrA fue considerada la causa de la resistencia a ácido nalidíxico ${ }^{(17,18)}$. Este cambio aminoacídico se asocia a una menor alteración de los patrones de hidrofobicidad de GyrA que otros cambios aminoacídicos en esa misma posición como por ejemplo $\operatorname{Ser}_{83} \mathrm{Leu}_{\mathrm{o}} \mathrm{Ser}_{83} \mathrm{Val}_{\text {, y }}$ por ende se suele asociar con menores niveles de resistencia ${ }^{(17,18)}$.

En adición a tetraciclina, Tet(A) se ha asociado a leves incrementos de resistencia a tigeciclina, más aún, se ha constatado el desarrollo durante tratamientos de nuevas variantes alélicas (cambio aminoacídico Ser ${ }_{251}$ Ala) capaces de conferir niveles de concentración mínima inhibitoria de $32 \mathrm{mg} / \mathrm{L}^{(19)}$.

Los integrones son estructuras genéticas movilizables, aunque no móviles per se, que se caracterizan por poseer un extremo 5 ' consistente en un gen codificante para una integrasa, una serie de casetes génicos codificantes para genes de diversa índole y un extremo 3' que varía entre los diferentes tipos de integrones, pero que dentro de ellos está altamente conservado ${ }^{(20)}$. Los integrones se clasifican en función del gen de la integrasa, siendo las clases 1 y 2 las más habituales, usualmente portando genes de resistencia ${ }^{(20)}$, y dentro de ellas reciben una numeración específica en base a su contenido (tipo y orden) de casetes génicos (http://integrall.bio. ua.pt/). El integrón detectado en la presente cepa es un integrón de clase 2 que contiene el gen $\operatorname{dfr} A 1$, concordante con el integrón In2-2 (http://integrall.bio.ua.pt/). Este gen $d f r A 1$, subyace bajo la resistencia a trimetoprim, y conjuntamente con el gen sul3, responsable de la resistencia a sulfisoxazol, bajo la resistencia a cotrimoxazol.

El estudio completo de esta cepa y la secuenciación de bla $a_{\text {TEM-176 }}$ se desarrolló en 2010, por lo que el tiempo transcurrido es la mayor limitación de este estudio, habiendo dado lugar a la paradoja que existan publicaciones donde se reporta la presencia de $b l a_{\mathrm{TEM}-176}$ previas al reporte original de esta $\beta$-lactamasa. Asimismo, queda pendiente evaluar el efecto real de AMC y el potencial como IRT de TEM-176, lo que debería hacerse clonando este gen en un vector de clonación que no contuviese otra $\beta$-lactamasa, para evitar interpretaciones erróneas debidas a la suma de la acción de ambas enzimas.

En conclusión, se caracterizan los mecanismos de resistencia a antimicrobianos presentes en la cepa D7111, reportándose la descripción original de TEM-176. Asimismo, se subraya la detección de la misma en estudios posteriores desarrollados en Perú, sugiriendo la presencia estable de este determinante de resistencia en el área.

Contribuciones de los autores: Todos los autores participaron en la concepción y diseño del artículo. MJP y SM participaron en el desarrollo experimental; análisis e interpretación de datos. JR y MJP participaron en la redacción del artículo. Todos los autores realizaron la revisión crítica del artículo, aprobaron la versión final y asumen responsabilidad de los contenidos del manuscrito.

Financiamiento: JR fue financiado por el Fondo Nacional de Desarrollo Científico, Tecnológico y de Innovación Tecnológica (FONDECYT - Perú) en el marco del «Proyecto de Mejoramiento y Ampliación de los Servicios del Sistema Nacional de Ciencia, Tecnología e Innovación Tecnológica» (número de contrato 08-2019-FONDECYT-BM-INC-INV).

Conflictos de interés: Los autores no declaran conflictos de interés.

\section{REFERENCIAS BIBLIOGRÁFICAS}

1. Ochoa TJ, Ruiz J, Molina M, del Valle LJ, Vargas M, Gil AI, et al. High frequency of antimicrobial drug resistance of diarrheagenic Escherichia coli in infants in Peru. Am J Trop Med Hyg. 2009;81:296-301.
2. Granda A, Riveros M, Martínez-Puchol S, Ocampo K, Laureano-Adame L, Corujo A, et al. Presence of extended-spectrum $\beta$-lactamase, CTX-M-65, in Salmonella enterica serovar Infantis isolated from children 
with diarrhea in Lima, Peru. J Pediatric Infect. Dis. 2019;14:194-200. doi: 10.1055/s-0039-1685502.

3. Palma N, Pons MJ, Gomes C, Mateu J, Riveros M, García W, et al. Resistance to quinolones, cephalosporins and macrolides in Escherichia coli causing bacteraemia in Peruvian children. J Global Antimicrob Resist. 2017;11:28-33. doi: 10.1016/j.jgar.2017.06.011.

4. Clinical and Laboratory Standards Institute. Performance Standards for Antimicrobial Susceptibility Testing, Twentieth Information Supplement M100S-16. Wayne: CLSI; 2010.

5. de Toro M, Seral C, Rojo-Bezares B, Torres C, Castillo FJ, Sáenz Y. Resistencia a antibióticos y factores de virulencia en aislados clínicos de Salmonella enterica. Enferm Infecc Microbiol Clin. 2014;32:4-10. doi: 10.1016/j.eimc.2013.03.006

6. Sáenz Y, Briñas L, Domínguez E, Ruiz J, Zarazaga M, Vila J, et al. Mechanisms of resistance in multiple-antibiotic-resistant Escherichia coli strains of human, animal, and food origins. Antimicrob Agents Chemother. 2004;48:3996-4001. doi: 10.1128/AAC.48.10.3996-4001.2004.

7. Clermont $\mathrm{O}$, Bonacorsi S, Bingen E. Rapid and simple determination of the Escherichia coli phylogenetic group. Appl Environ Microbiol. 2000;66:4555-8. doi: 10.1128/aem.66.10.4555-4558.2000.

8. Datta N, Kontomichalou P. Penicillinase synthesis controlled by infectious R factors in Enterobacteriaceae. Nature. 1965;208:239-41. doi: 10.1038/208239a0.

9. Desvars-Larrive A, Ruppitsch W, Lepuschitz S, Szostak MP, Spergser J, Feßler AT, et al. Urban brown rats (Rattus norvegicus) as possible source of multidrug-resistant Enterobacteriaceae and meticillin-resistant Staphylococcus spp., Vienna, Austria, 2016 and 2017. Euro Surveill. 2019;24:1900149. doi: 10.2807/1560-7917.ES.2019.24.32.1900149.

10. Dobiasova H, Dolejska M. Prevalence and diversity of IncX plasmids carrying fluoroquinolone and $\beta$-lactam resistance genes in Escherichia coli originating from diverse sources and geographical areas. J Antimicrob Chemother. 2016;71:2118-24. doi: 10.1093/jac/dkw144.
11. Guo S, Tay MYF, Thu AK, Seow KLG, Zhong Y, Ng LC, et al. Conjugative IncX1 plasmid harboring colistin resistance gene mcr-5.1 in Escherichia coli isolated from chicken rice retailed in Singapore. Antimicrob Agents Chemother. 2019;63:e01043-19. doi: 10.1128/AAC.01043-19.

12. Mshana SE, Hain T, Domann E, Lyamuya EF, Chakraborty T, Imirzalioglu C. Predominance of Klebsiella pneumoniae ST14 carrying CTX-M-15 causing neonatal sepsis in Tanzania. BMC Infect Dis. 2013;13:466. doi: 10.1186/1471-2334-13-466.

13. Vinueza-Burgos C, Ortega-Paredes D, Narváez C, De Zutter L, Zurita J. Characterization of cefotaxime resistant Escherichia coli isolated from broiler farms in Ecuador. PLoS One. 2019;14:e0207567. doi: 10.1371/ journal.pone.0207567.

14. Pehrsson EC, Tsukayama P, Patel S, Mejía-Bautista M, Sosa-Soto G, Navarrete $\mathrm{KM}$, et al. Interconnected microbiomes and resistomes in low-income human habitats. Nature. 2016;533:212-6. doi: 10.1038/nature17672.

15. Stapleton P, Wu PJ, King A, Shannon K,French G, Philips I. Incidence and mechanisms of resistance to the combination of amoxicillin and clavulanate in Escherichia coli. Antimicrob Agents Chemother. 1995;39: 2478-83.

16. Goñi MP, Agudo MC, Gómez-Lus R. Antibiòtics aminoglicòsids. Treb Soc Cat Biol. 2004;55:107-19.

17. Ruiz J. Mechanisms of resistance to quinolones: target alterations, decreased accumulation and DNA gyrase protection. J Antimicrob Chemother. 2003;51:1109-17. doi: 10.1093/jac/dkg222.

18. Ruiz J. Quinolones: acció i resistencia. Treb Soc Cat Biol. 2004; 55: 131-8.

19. Du X, He F, Shi Q, Zhao F, Xu J, Fu Y, et al. The rapid emergence of tigecycline resistance in $b l a_{\mathrm{KPC}-2}$ harboring Klebsiella pneumoniae, as mediated in vivo by mutation in tetA during tigecycline treatment. Front Microbiol. 2018;9:648. doi: 10.3389/fmicb.2018.00648.

20. Li Y, Yang L, Fu J, Yan M, Chen D, Zhang L. Genotyping and high flux sequencing of the bacterial pathogenic elements - integrons. Microb Pathog. 2018;116:22-5. doi: 10.1016/j.micpath.2017.12.073. 